\title{
Las redes sociales y su efecto sobre la confianza en las tiendas online
}

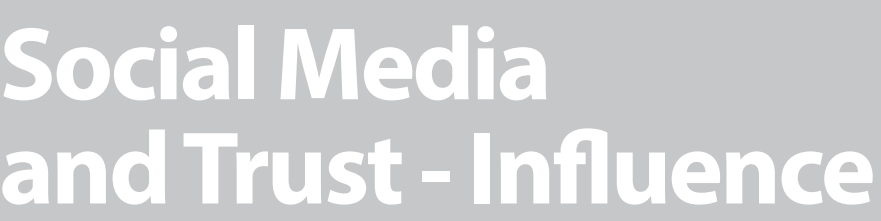
on e-Tailers

Juan Carlos Higueras Redecillas ESIC Marketing \& Business School juancarlos.higueras@esic.edu Julio Alard Josemaría ESIC Marketing \& Business School julio.alard@esic.edu

Carmelo Mercado Idoeta Universidad Rey Juan Carlos carmelo.mercado@urjc.es
Higueras Redecillas, J.C., Alard Josemaría, J., Mercado Idoeta, C. (2016)

Las redes sociales y su efecto sobre la confianza en las tiendas online

Revista Internacional de Investigación en Comunicación aDResearch ESIC. No $14 \mathrm{Vol} 14$

Segundo semestre, Julio-Diciembre 2016 · Págs. 30 a 53 DOI: 14.7263/ADRESIC.014.002 
RESUMEN

Clasificación JEL:

M31

Palabras clave:

confianza,

notoriedad,

imagen,

calidad de servicio,

tienda online,

redes sociales,

SEM

ABSTRACT

JEL Classification:

M31

Key words:

trust,

awareness,

image,

quality of service,

online store,

social media,

SEM
El comercio electrónico está creciendo a elevadas tasas y, con ello, están apareciendo multitud de tiendas online que buscan posicionarse y diferenciarse en el mercado global. Por otra parte, la presencia de las empresas en los medios sociales y el desarrollo de comunidades alrededor de la marca corporativa de los comerciantes minoristas, está favoreciendo la compartición de opiniones y experiencias entre los individuos, lo que genera mayores niveles de notoriedad y una imagen percibida de las tiendas online que es compartida por los consumidores que acceden a las diferentes plataformas de las redes sociales. Igualmente, el boca oreja online tiene un efecto sobre la confianza que los consumidores depositan en una tienda virtual, algo que cada vez es más relevante para conseguir la diferenciación. En este artículo se estudia la relación entre las actividades en medios sociales de la tienda y la generación de confianza, a través de la notoriedad, la imagen percibida y la calidad del servicio online como variables mediadoras. Para ello, se realizó una investigación de mercado sobre 301 individuos mediante un cuestionario online auto-administrado. Los resultados del modelo de ecuaciones estructurales mostraron evidencia del efecto de los medios sociales sobre la notoriedad y la imagen percibida de la tienda, no encontrándose evidencia del efecto sobre la calidad percibida. Igualmente, se encontró evidencia de la relación existente entre la imagen de la tienda y la calidad del servicio sobre la confianza online.

E-commerce is growing at high rates, hence quite a lot of retailers are appearing in the Internet, trying to make a difference in the global market. Moreover, the presence of many companies in social media platforms and the development of virtual communities around the retailer corporate brand, are encouraging the sharing of reviews and experiences between consumers, creating higher levels of retailer awareness and a perceived image for online stores that is shared by consumers who access social networks. In addition, online word of mouth has an influence on the trust that consumers place in a virtual store, something that is increasingly important for earning a competitive edge. This article discusses the relationship between the retailers' social media activities and the process for building trust through awareness, the perceived image and quality of the online service as mediating variables. For this, a survey of 301 consumers was conducted using a self-administered online questionnaire. The findings of the structural equation model showed evidence of the effect of social media on awareness and perceived image of the store, finding no evidence of any influence on perceived quality of service. Likewise, evidence of the relationship between online store image and quality of service in retailer trust was also found. 


\section{Introducción}

En el presente trabajo, se aborda el efecto que tienen los medios sociales sobre la confianza de las tiendas online, utilizando algunas de las principales dimensiones del capital marca, tales como la notoriedad, la imagen de la tienda y la calidad del servicio ofrecido como factores mediadores.

En concreto, se buscaba analizar si los medios sociales ejercen un efecto directo y positivo sobre la imagen percibida por el consumidor de las tiendas online; sobre la notoriedad de las mismas, y sobre la calidad percibida que el consumidor tiene de la tienda online.

Para ello, se desarrolló una investigación de mercado sobre 301 individuos mediante un cuestionario online auto-administrado. Los resultados del modelo de ecuaciones estructurales, tal y como se exponen al final del trabajo, mostraron evidencia del efecto de los medios sociales sobre la notoriedad y la imagen percibida de la tienda, no encontrándose evidencia del efecto sobre la calidad percibida. Igualmente, se encontró evidencia de la relación existente entre la imagen de la tienda y la calidad del servicio sobre la confianza online.

En este sentido, tal y como afirman Goode y Harris (2007), la confianza es uno de los factores clave que estimula la compra online y juega un papel importante sobre la capacidad que tienen los consumidores para dar credibilidad a alguien que comparte cualquier tipo de información, ya sea una opinión o una experiencia de compra.

Por otra parte, los medios sociales se han convertido en uno de los elementos que forman parte de la vida diaria de numerosas personas, que pasan cada vez más tiempo conectados a plataformas de redes sociales para compartir comentarios, opiniones y experiencias (Ayestarán, 2014) de modo que, de una manera más importante los contenidos de los medios sociales, influyen en el comportamiento y las percepciones de los individuos, desde el proceso inicial de búsqueda de información hasta la postventa (Laroche et al., 2012), lo que junto al desarrollo de Internet está modificando los mecanismos con los que se relacionan las empresas y los clientes (Rowley, 2006).

A la vista de lo anterior, la mayoría de las compañías muestran, cada vez, mayor interés en tener presencia en las redes sociales y en desarrollar comunidades, alrededor de su marca corporativa, ya que, tal como indican Kaplan y Haenlein (2010), los medios sociales gozan de grandes ventajas al permitir conectar a las compañías con los consumidores con un coste bajo y de forma rápida.

Además, cabe señalar que el proceso de digitalización de la sociedad ha traído grandes transformaciones entre las que destaca la de la comunicación comercial. En este sentido, Internet, tal y como apunta Silverstein (2002), se ha convertido en un medio de comunicación muy poderoso, ya que consigue reunir tanto a compradores como vendedores, de cualquier lugar del mundo, en un mismo espacio virtual, generando una nueva forma de publicitar la oferta comercial de las compañías.

Castelló (2010), por su parte señala que Internet ofrece, como medio de comunicación, la posibilidad de generar interactividad y mayor involucración de los clientes, en especial mediante la creación de comunidades virtuales y su efecto complementario a otros mecanismos publicitarios, ya que posibilita el uso de una amplia gama de formatos y soportes.

A pesar de esto, las tiendas online no están desarrollando el potencial comercial que pueden tener sus sitios web. Tal y como sugieren Zhang 
et al. (2010), nos encontramos en fases tempranas en cuanto al aprovechamiento de las posibilidades que ofrece Internet para las compañías, por ello, la mayoría de las empresas, utilizan sus sitios web como herramienta de comunicación más que como mecanismos de estimulación comercial.

De acuerdo con Park et al. (2007), los consumidores confían cada vez más en las opiniones de otros consumidores en Internet, mientras que los mecanismos tradicionales de promoción y publicidad comercial están decreciendo en efectividad (Sethuraman et al., 2011).

En este sentido, los consumidores están modificando sus hábitos de búsqueda de información sobre productos y servicios, de modo que cada vez más se apoyan en el boca oreja electróni$\mathrm{CO}^{1}$, mediante el acceso a las redes sociales o a los sitios de análisis de prestaciones de productos y servicios (Gruen et al., 2006; Dijkmans et al., 2015). En esta línea, tal y como indica De Salas (2002), los tradicionales formatos de comunicación publicitaria están perdiendo su eficacia en favor de nuevos mecanismos que integran diversos contenidos interactivos y que favorecen la comunicación más personalizada entre las empresas y los clientes, de modo que, aparece una nueva dimensión del proceso de comunicación, basada en la interactividad.

Uno de los principales objetivos del marketing empresarial es establecer diferentes mecanismos de comunicación mediante los que una compañía puede informar a los clientes sobre su oferta de productos y servicios, generando atractivo por su oferta. En este sentido, generar un sentimiento de compromiso, en los medios sociales, hacia la marca corporativa, ayuda a gene-

1 eWord Of Mouth en terminología anglosajona rar relaciones con los clientes y aumentar los beneficios de la empresa (Kim y Ko, 2012).

Así pues, el desarrollo reciente del comercio electrónico y los medios sociales, y la escasez de literatura específica, hace necesario un replanteamiento, tanto por profesionales como académicos, de las cuestiones del comercio tradicional, ya que la aparición de los nuevos modelos de comercio y los nuevos usos del mundo digital hace necesario el estudio del comportamiento de los consumidores y de los nuevos canales de comercio electrónico, en aspectos clave para la gestión de las marcas.

Este trabajo se estructura de la siguiente forma. En el punto 2, se realiza una revisión de la literatura, donde se analizan los aspectos más relevantes del comercio electrónico y su rápido crecimiento, a nivel nacional e internacional. Además se recoge las principales relaciones a nivel teórico que se han encontrado entre medios sociales, notoriedad, imagen, calidad de servicio y confianza en las tiendas online. A partir de este marco teórico se formulan las principales hipótesis que se han tenido en cuenta para su posterior contraste. En el apartado 3 se aborda la metodología utilizada para el contraste de hipótesis. El punto 4 está dedicado al análisis de los principales resultados del estudio, y por último en el apartado 5 se recogen las principales conclusiones del estudio, así como futuras líneas de investigación en esta área.

\section{Revisión de la literatura Comercio electrónico}

El comercio electrónico, en los últimos años, está viviendo una importante transformación y desarrollo a nivel mundial, facilitando a los consumidores un entorno cómodo para realizar sus compras (Chen, 2012), a la vez que tiene un 
gran potencial como herramienta de marketing (Liberos et al., 2010). Todo ello, año tras año, está favoreciendo el crecimiento de las ventas online (Kim y Forsythe, 2010; Prado et al., 2014), lo que se pone de manifiesto a través de numerosos informes sectoriales.

A nivel mundial, las estimaciones de eMarketer (2014) indican que las ventas de comercio electrónico minorista alcanzarán los 2.357 billones de euros en el año 2017, lo que refleja una tasa media anual de crecimiento del $24,5 \%$, considerando que dichas ventas fueron de 1.058 billones de euros en el año 2012.

A nivel nacional, de acuerdo con el informe de ONTSI (2015), las compras B2C en España alcanzaron un valor de 16.259 millones de euros en el año 2014 frente a los 14.610 millones euros del año anterior, lo que supone un incremento del 11,3\%. Igualmente, en España, de acuerdo con CNMC (2016)², el comercio electrónico alcanzó, en el tercer trimestre de 2015, un volumen de negocio de 5.302,6 millones de euros, lo que supone una tasa de crecimiento interanual del 29,2\%. Asimismo, se registraron, en dicho periodo, un $27,4 \%$ más de transacciones, alcanzando un total de 74 millones de transacciones. Por sectores, las actividades que mayores ventas han tenido son los relacionados con el turismo (20,8\%), el transporte aéreo (13,0\%), la ropa y complementos (5,6\%) y marketing directo (4,9\%).

En cuanto a las compras realizadas por los consumidores españoles en tiendas online nacionales, aumentaron un $24,9 \%$ en términos interanuales hasta alcanzar un volumen de 2182,2 millones de euros. Asimismo, de acuerdo con ONTSI (2015), el gasto medio anual por com-

2 https://www.cnmc.es/Portals/0/Ficheros/notasdeprensa/2016/ TelecomunicacionesAudiovisual/20160401_NP_CE_III_15_.pdf consultado el 21/04/2016 prador, en el año 2014, había crecido un 3,3\% respecto del año anterior, situándose en $876 €$, en línea con lo que sugieren Arce y Cebollada (2011) en términos de cambios en los hábitos de compra del consumidor que ha incrementado el valor per cápita de la compras en internet.

Comprar online se ha convertido en una de las actividades más populares en Internet (Doherty et al., 2003; Forsythe y Shi, 2003), lo que, junto a otros factores, está estimulando el crecimiento del número de tiendas online.

Yoo y Donthu (2001) indican que una tienda virtual es la versión online de una tienda física en la que los procesos tienen lugar en el ciberespacio. Asimismo, definen la tienda online como un sitio web de comercio minorista en el que los consumidores pueden visualizar, evaluar y comprar un producto o servicio. En este contexto, los minoristas tradicionales deben hacer frente a una serie de retos para sobrevivir en el nuevo escenario (Neslin et al., 2006), entre los que destacan el desarrollo de una estrategia multicanal que les permita aprovechar las sinergias entre las tiendas físicas y las virtuales, identificando aquellos canales que, de acuerdo con las preferencias del consumidor, son los más adecuados para competir (Hsieh et al., 2012; Pantano y Viassone, 2015).

Así pues, desde el punto de vista del consumidor, el comercio electrónico permite acceder a un mayor número de tiendas con una oferta más amplia, con precios más atractivos y con la posibilidad de poder comprar desde cualquier lugar a cualquier hora, recibiendo el pedido en casa y facilitando un mayor grado de comodidad en el proceso de compra (Quelch y Klein, 1996; Balaghi, 2014). Por otro lado, desde la perspectiva de la empresa, permite crear un canal adicional para hacer negocio y acceder a nuevos mercados 
debido a la disminución de las barreras a la entrada, reduciendo el poder de los establecimientos tradicionales (Jarvenpaa et al., 2000), todo ello sin la necesidad de desarrollar nuevas infraestructuras físicas (Rodríguez, 2008).

\section{Confianza y tiendas online}

De acuerdo con Urban et al. (2000), la confianza es la moneda de cambio en las compras online lo que influye en el comportamiento de los consumidores y la intención de compra (Lee y Lin, 2005). Por ello, la falta de confianza es una de las principales barreras por la que muchos consumidores no compran online.

Por otra parte, la confianza es considerada como un concepto multidimensional y complejo que es difícil conceptualizar y medir. La falta de consenso al respecto ha provocado que haya una gran diversidad de definiciones, lo que dificulta tanto su comprensión como la identificación de sus dimensiones a nivel conceptual y empírico. En esta línea, numerosos estudios consideran la confianza, bien como un constructo unidimensional (Jarvenpaa et al., 2000; Gefen et al., 2003; Pavlou (2003); Bart et al., 2005; Flavián y Guinaliu, 2006; Casalo et al., 2007) o multidimensional (Gefen y Straub, 2004; Wu y Chang, 2006).

Asimismo, la confianza es uno de factores más importantes para el crecimiento del comercio electrónico, en general, y para el aumento de las compras de una tienda online, en especial cuando se encuentra en las fases tempranas de lanzamiento comercial (Jarvenpaa et al., 2000). Además, las compras online, desde la perspectiva de la tienda, son realizadas por procesos electrónicos y no por personas, por ello, la confianza se convierte en una creencia subjetiva del consumidor acerca de que dicha tienda virtual cumplirá con todas sus obligaciones (Kim et al., 2009).

Por otro lado, la falta de seguridad percibida por los compradores online representa un riesgo y uno de los principales frenos para el desarrollo del comercio electrónico (Dong-Her et al., 2004). En esta línea, el incremento de los robos de datos de clientes en conocidas tiendas online, pone de manifiesto que, para generar confianza, los minoristas virtuales deben poder garantizar, tanto la seguridad del sitio web como la confidencialidad de la información de los clientes (Martínez et al., 2006).

En la misma línea, la confianza es un factor que favorece las relaciones comerciales estables si bien, la falta de mecanismos de compra tan consolidados como en los mercados tradicionales, genera un menor grado de disposición de los consumidores hacia la compra online, ya que es más difícil generar un sentimiento de confianza hacia la tienda virtual (Prado et al., 2014). Así pues, para que exista confianza, la tienda online debe gozar de credibilidad, de modo que los consumidores tengan expectativas positivas acerca del intercambio que se debe realizar en adecuadas condiciones de seguridad y fiabilidad (Gil-Saura et al., 2013).

Aunque la confianza es un factor que interviene en las diferentes fases de la decisión de compra, tanto en la etapa de búsqueda de información como de compra online, en esta investigación, se ha considerado sólo esta última etapa. Por tanto, la medida de la confianza en la tienda online vendrá dada por aspectos de confianza general, de credibilidad en términos de cumplimiento de los compromisos que indica el sitio web, así como garantía de confidencialidad de los datos de los clientes y seguridad en la transacción electrónica. 


\section{Medios sociales y tiendas online}

Los medios sociales son un conjunto de aplicaciones basadas en Internet que se construyen sobre los pilares ideológicos y tecnológicos de la Web 2.0 y que permiten la creación y el intercambio de contenidos generados por el usuario (Kaplan y Haenlein, 2010), superando los límites que impone el tiempo y la distancia, siendo uno de sus principales beneficios la compartición de experiencias de compra (Bazarova, 2016), la comunicación con la marca y la realización de revisiones de productos o servicios de forma cómoda y rápida para los consumidores.

En la misma línea, los medios sociales, de acuerdo con Strater y Richter (2007) son aplicaciones online, plataformas y medios cuyo objetivo es facilitar las interacciones, colaboraciones y la compartición de contenidos, pudiendo tener diferentes formas, tales como weblogs, social blogs, wikis, microblogging, podcasts, fotos o videos, entre otros (Mangold y Faulds, 2009; Kim y Ko, 2012), siendo los más populares Facebook, Twitter, Youtube e Instagram (Laroche et al., 2013).

Así pues, en el nuevo contexto de Internet, donde las redes sociales juegan un papel muy relevante, los consumidores son menos sensibles a las estrategias de comunicación personalizadas, ya que el boca oreja es más eficaz que aquéllas para determinar actitudes y comportamientos de compra, basados en opiniones sobre los productos (Brown et al., 2007; Trusov et al., 2009).

Por otra parte, cada día es mayor el número de usuarios de Internet que utilizan las redes sociales tales como Facebook o Twitter, ya sea para consultar o compartir información sobre productos y/o servicios, para contactar con otras personas o simplemente por ocio (Kaplan y
Haenlein, 2010), de modo que, las tiendas online, a la vista de estos comportamientos, comienzan a tener mayor presencia en las redes sociales para mejorar sus cifras de ventas, ya sea por incremento en el tráfico de clientes hacia el sitio web, o bien para incrementar la satisfacción y la lealtad de sus clientes, así como la notoriedad (De Vries et al., 2012) y la imagen del negocio o de su marca (Kietzmann et al., 2011) o crear valor gracias a un mejor servicio postventa al conocer mejor lo que realmente interesa a los consumidores (Golob et al., 2013). Todo ello, puede favorecer una mejor relación de la compañía con su público objetivo, lo que se traduce en un mecanismo eficaz para generar marca y romper la desconfianza ante la empresa.

Asimismo, son numerosas las investigaciones que estudian la influencia de los medios sociales sobre los diferentes aspectos del comportamiento del consumidor (e.g. Mangold y Faulds, 2009; Kim y Ko, 2012; Laroche et al., 2013; Ioanăs y Stoica, 2014; Tresna y Wijaya, 2015).

Otro de los factores que favorecen el uso de los medios sociales es la necesidad, cada vez mayor, de los consumidores por ahorrar tiempo, evitando tediosos procesos de búsqueda de información, para lo que acceden a los comentarios y experiencias de otros consumidores (Brown et al., 2007), lo que en algunos casos puede ayudar a tomar las decisiones de compra (Kim y Ko, 2012) y, en este sentido, el boca oreja electrónico tiene un importante impacto sobre el comportamiento del consumidor (Zhu y Zhang, 2010), pues, en términos de credibilidad, el consumidor confía más, en las opiniones de terceros con los que tiene una relación cercana, tales como familiares y amigos, que en la de otros con los que mantienen vínculos más débiles (Cheung et al., 2008). 
Por otra parte, mientras que algunos consumidores utilizan los medios sociales de forma pasiva, para obtener recomendaciones o información de terceros, otros lo hacen de forma activa para generar contenidos y opiniones, al igual que otros que son tanto consumidores como generadores del boca oreja electrónico (Park y Fairhurst, 2010; López y Sicilia, 2013). Por ello, en esta investigación se han considerado los medios sociales en sentido amplio, no se ha limitado sólo a las redes sociales, sino a cualquier otro medio que permita el intercambio entre consumidores, a través de Internet, de contenido sobre tiendas online.

\section{Medios Sociales, Notoriedad, Imagen y Calidad de Servicio}

La compartición de información y experiencias entre los miembros de una comunidad virtual, junto con la involucración de los responsables de las tiendas online, permite reforzar los vínculos entre la comunidad y la compañía (Laroche et al., 2013), a la vez que dichas opiniones pueden ser un mecanismo adecuado para medir la reputación de la tienda (Serrano-Cinca et al., 2010). De esta forma, que cuanto mayor en la intensidad de uso de las redes sociales por parte de los consumidores de una compañía, mayor es su efecto sobre la reputación (Dijkmans et al., 2015).

Igualmente, de acuerdo con Holland y Baker (2001) las comunidades virtuales son una importante fuente de boca oreja online que permiten que los consumidores, al otorgar mayor valor a las recomendaciones de dichas comunidades, compartan y generen determinadas asociaciones comunes hacia la tienda.

Por otro lado, los medios sociales ejercen un gran efecto sobre la notoriedad de las compañías. Tal y como afirman Mangold y Faulds (2009), el comportamiento de los consumidores, en términos de notoriedad, se ve influenciado por el crecimiento de los mensajes y evaluaciones de productos o servicios en los medios sociales. Por otro lado, son numerosos los estudios sobre el boca oreja que muestran la relación entre el uso de los medios sociales y la notoriedad de las empresas (e.g. Drèze y Zufryden, 2004; Dellarocas y Narayan, 2006; Serrano-Cinca et al., 2010; Fanion, 2011; Marsden, 2011; Kotler y Keller, 2012; Haque et al., 2013; Shojaee y Azman., 2013; Tsimonis y Dimitriadis, 2014).

En esta línea, Gunelius (2011) afirma que el intercambio de información que los consumidores realizan a través de las redes sociales, permiten incrementar la notoriedad con un coste inferior para las compañías. De igual forma, los medios sociales son una herramienta que favorece la notoriedad de las empresas, al poder llegar a millones de personas, de forma fácil (Fanion, 2011), pues la presencia de una tienda en dichos medios, permite que los consumidores se familiaricen con la misma (Tsimonis y Dimitriadis, 2014) mejorando los flujos de comunicación con la empresa (Zhu y Zhang, 2010).

Por lo tanto, los medios sociales, como mecanismos para informar sobre nuevos productos o servicios, mediante foros de opinión, son herramientas útiles, populares y eficaces que permiten acelerar la notoriedad (Marsden, 2011). En este sentido, tal y como indican Tsimonis y Dimitriadis (2014), uno de los beneficios de los medios sociales buscados por los directivos de las empresas es el rápido aumento en la notoriedad de la compañía, por la viralidad de los mensajes de los consumidores que se favorece por el boca oreja a traves de dichos medios.

Asimismo, la existencia de comunidades virtuales y de foros de opinión, en donde se com- 
parte la información de los consumidores, pueden ser un buen mecanismo para evaluar la popularidad de un sitio web (Serrano-Cinca et al., 2010), al permitir a los comerciantes minoristas acceder a los mismos y ofrecer dichos comentarios a los clientes.

Por otro lado, dada la intangibilidad del servicio que ofrece una tienda online, la evaluación de su calidad percibida es compleja y, por ello, el cliente utiliza los comentarios recogidos en los medios sociales como una señal de calidad del servicio (Berry et al., 2002) generando unas expectativas que se basan en las experiencias de otros consumidores (Kotler y Keller, 2012).

En la medida en que los mensajes, opiniones y experiencias vertidas en los medios sociales, sean creíbles, la percepción de la calidad del servicio se verá influenciada por ellos, ya que dichos comentarios serán más persuasivos si el cliente considera que la credibilidad de la fuente es elevada (Cheung et al., 2008; López y Sicilia, 2013), lo que ocurre especialmente en las redes sociales de las enseñas comerciales ya que se generan comunidades virtuales de marca, las cuales suelen generar mayor credibilidad al consumidor que tenga un sentimiento de pertenencia hacia dicho grupo social (Dijkmans et al., 2015).

A la vista de todo lo anterior, se establecen las siguientes hipótesis:

- H1: Los medios sociales ejercen un efecto directo y positivo sobre la imagen percibida por el consumidor de las tiendas online

- H2: Los medios sociales ejercen un efecto directo y positivo sobre la notoriedad de las tiendas online

- H3: Los medios sociales ejercen un efecto directo y positivo sobre la cali-

\section{dad de servicio percibida por el consu- midor de la tienda online}

\section{Notoriedad y tiendas online}

Aaker (1996) define la notoriedad de la marca como «la capacidad que tiene un cliente potencial de reconocer o recordar una marca», lo que guarda relación con el tiempo que permanece la marca en la mente del consumidor, por tanto, la notoriedad de una marca tiene tres niveles, el reconocimiento, el recuerdo y el posicionamiento ${ }^{3}$ de la marca en la mente del consumidor. Igualmente, Pappu y Quester (2006) definen la notoriedad de una tienda como «la capacidad que tiene el consumidor para reconocer o recordar esa tienda dentro de una determinada categoría».

Tal y como indican Hartman y Spiro (2005), la notoriedad de un establecimiento está asociada a su identidad y se define como el nombre y/o logo asociado a la tienda cuyo conocimiento, por parte del consumidor, puede influenciar el proceso de recuerdo o reconocimiento de dicha tienda.

Ahora bien, en Internet, la notoriedad de una tienda online puede ser igual de importante que lo es para una tienda convencional (Page y Lepkowska-White, 2002), por ello, los minoristas virtuales consumen muchos recursos para conseguir las mejores posiciones en buscadores y portales y, además, una de las actividades favoritas de ocio de muchos consumidores consiste en navegar por Internet para leer y aportar comentarios en foros y blogs (Serrano-Cinca et al., 2010). Por ello, la notoriedad de una tienda online juega un papel importante ya que cuanto mayor sea, más probable será que un consumi-

\footnotetext{
3 Aaker hace referencia al Top of Mind, es decir, si la marca es la primera en ser recordada cuando se pide a una persona que nombre marcas de una determinada categoría.
} 
dor, en un proceso de búsqueda, explore en primer lugar el sitio web de dicha tienda.

\section{Notoriedad, Imagen y Calidad}

De acuerdo con Keller (1993), un consumidor debe conocer una marca antes de poder desarrollar una imagen de la misma, por ello, la notoriedad es una dimensión que debe preceder a la imagen de marca. Igualmente, otros autores, sugieren que existe una fuerte relación entre la notoriedad y la imagen percibida (Pappu et al., 2005) e incluso pueden ser consideradas una única dimensión difícil de separar (Yoo et al., 2000).

De acuerdo con Hartman y Spiro (2005), el conocimiento de un distribuidor minorista consiste en un conjunto de asociaciones, que se encuentran en algún lugar de la mente del consumidor, relacionadas con el nombre de la tienda y que proporciona información para desarrollar una impresión general sobre dicho establecimiento, creada a partir de asociaciones hacia la tienda.

Numerosos autores de la literatura han realizado investigaciones empíricas donde se muestra la relación existente entre la notoriedad de un distribuidor minorista y la imagen que perciben los consumidores (Villarejo-Ramos et al., 2007). De igual forma, diversos estudios (e.g. Wang y Hwang, 2001; Khan et al., 2015) han mostrado la relación existente entre la notoriedad de una marca y la calidad percibida del producto o servicio comercializado.

A la vista de todo lo anterior se proponen las siguientes hipótesis:

- H4: La notoriedad de una tienda online ejerce un efecto directo y positivo sobre la imagen percibida por el consumidor

- H5: La notoriedad de una tienda online ejerce un efecto directo y positivo sobre la calidad del servicio percibida por el consumidor

\section{Imagen de la tienda online}

La imagen que el consumidor percibe de un distribuidor minorista, puede determinar su intención de compra (Wu et al., 2013) así como sus comportamientos y conductas (Grewal et al., 2003).

Varios estudios han investigado la imagen de una tienda (Hartman y Spiro, 2005; Martenson, 2007), en términos del conjunto de percepciones del consumidor, basadas en diferentes características funcionales o atributos psicológicos, tales como el servicio, la gama, la conveniencia, el ambiente de la tienda y la calidad (Grewal et al., 2003), lo que posibilita tener una impresión global del distribuidor minorista.

En el caso de las tiendas online, de acuerdo con Serrano-Cinca et al. (2010), la reputación, juega un papel clave, pues los productos o servicios comercializados, no pueden ser percibidos con los cinco sentidos, de modo que las opiniones y recomendaciones que comparten los consumidores tienen un gran valor. Por tanto, el consumidor establece diferentes asociaciones acerca de un establecimiento minorista, si bien existen dos más relevantes, una relativa a la imagen que percibe de la tienda o de su marca corporativa y, otra, basada en la reputación del minorista.

\section{Imagen de La Tienda Online, Calidad de Servicio y Confianza}

A lo largo de la literatura sobre sobre establecimientos comerciales, existen numerosos estudios que muestran la relación existente entre las asociaciones que el consumidor desarrolla en su mente y los niveles de calidad percibida que acumula (Dick et al., 1995; Grewal et al., 2003; Martenson, 2007; Beristain y Zorrilla, 2011). 
Por otro lado, la imagen de un establecimiento contribuye a la percepción de calidad por parte de los consumidores, aumentando sus expectativas (Yoo et al., 2000; Villarejo-Ramos et al., 2007). En este sentido, la imagen de marca corporativa del establecimiento actúa como una señal o indicador de la calidad (Dick et al., 1995) pues los consumidores, además de identificar la categoría de productos, pueden desarrollar percepciones de calidad reduciendo el riesgo asociado a la compra (Kotler y Keller, 2012), de modo que una imagen de marca positiva será asociada a una elevada calidad percibida, lo que afectará a la actitud y comportamiento del consumidor, condicionando sus decisiones de compra en base a dichas señales (Grewal et al., 2003).

Por otra parte, de acuerdo con Wolfinbarger y Gilly (2003), los consumidores que compran online, tienden a implicarse mucho más en el proceso de compra, lo que incrementará el desarrollo de asociaciones, opiniones o creencias, de tipo cognitivo, lo que, a su vez, influirá en la confianza que un consumidor manifieste acerca de la tienda online. Así pues, dada la incertidumbre que, para algunos consumidores, representa el comercio electrónico, éstos no realizan compras mientras no haya una disminución de sus incertidumbres y, para ello, tenderán a desarrollar previamente una evaluación del entorno online y una serie de creencias (Martínez et al., 2006).

En línea con lo anterior, Jarvenpaa et al. (2000) investigaron el efecto de la reputación de un comerciante minorista sobre la confianza del consumidor en la tienda, llegando a la conclusión de que existía una fuerte relación positiva y directa entre ambos conceptos. Así pues, a mayor reputación, mayor confianza, lo que facilita- rá atraer más clientes y realizar mayor volumen de ventas que otras tiendas similares.

A la vista de la revisión de los diferentes estudios e investigaciones de la literatura sobre imagen percibida de la tienda, se plantean las siguientes hipótesis:

- H6: La imagen percibida de la tienda online por el consumidor ejerce un efecto directo y positivo sobre la calidad percibida del servicio

- H7: La imagen percibida de la tienda online, por el consumidor ejerce un efecto directo y positivo sobre la confianza en la tienda

\section{Calidad del servicio y tiendas online}

El desarrollo del comercio electrónico junto con la aparición de numerosas tiendas online con modelos de negocio y productos similares, favorece que la calidad del servicio ofrecido se convierta en la única fuente de diferenciación y creación de valor para el cliente (Parasuraman et al., 1988; De Ruyter et al., 2001).

De acuerdo con Zeithaml et al. (2002), la calidad del servicio electrónico se define como el grado en que un sitio web facilita al consumidor que la compra, el pago y la entrega de los productos o servicios se realice de forma eficaz y eficiente $y$, por tanto, el servicio comienza cuando el cliente entra en la tienda y termina con la correcta entrega del producto.

Asimismo, dicha calidad de servicio percibida puede influenciar, según numerosos autores, sobre el comportamiento del consumidor (Constantinides, 2004; Vrechopoulos et al., 2004; Manganari et al., 2009), ya que, en el entorno online, unas tiendas se diferencian de otras no por los productos que comercializan sino por el servicio que ofrecen. 
Por otro lado, los entornos basados en internet permiten a las empresas identificar mejor las necesidades de los clientes, lo que permite mejorar la gestión del servicio ofrecido por la tienda y ello aumenta el número de visitantes así como mejora su atractivo. En esta línea, para muchos consumidores, tal como sugiere Aladwani (2006), puede ser más importante la experiencia asociada a la compra que el resultado final de la misma, de modo que la calidad percibida por el consumidor del servicio proporcionado por la tienda online, se convierte en un factor relevante (Zeithaml et al., 2002).

De la revisión de la literatura se concluye la falta de consenso, en la conceptualización de la calidad de servicio online y sus dimensiones, entre los diferentes autores. Si bien para algunos investigadores, el diseño y disponibilidad del sitio web son factores clave para medir la calidad del servicio online (Martínez et al., 2006), para una gran mayoría (e.g. Wolfinbarger y Gilly, 2003; Parasuraman et al., 2005), se trata de un conjunto de atributos entre los que destacan el atractivo del sitio web, su velocidad de descarga, el cumplimiento y fiabilidad, así como la capacidad de dar respuesta a las solicitudes del cliente.

\section{Calidad se servicio online y confianza}

Numerosas investigaciones empíricas muestran la influencia directa de la calidad del servicio electrónico sobre la confianza en la tienda online (Ribbink et al., 2004) sugiriendo que la confianza en la tienda online mejora mediante la puesta en marcha de políticas que mejoren la calidad del servicio electrónico ofrecido por el distribuidor minorista, ya sea en términos del cumplimiento de los compromisos, en general, (Kim et al., 2009), o bien aspectos concretos como la entrega en plazo de las compras o la garantía de devolu- ción y reembolso ante no conformidad del cliente. En cualquier caso, la confianza en la tienda virtual es un factor relevante en el proceso de compra online (Lee y Lin, 2005), pues la entrega del producto o servicio comprado es más difícil de conseguir en un establecimiento virtual que en uno convencional (Jarvenpaa et al., 2000).

A pesar de la diversidad de modelos de medida de la calidad de servicio electrónico, en esta investigación, de acuerdo con Collier y Bienstock (2006) y el modelo e-SERVQUAL de Zeithaml et al. (2002), se ha considerado que la escala de calidad de servicio online se descompone en tres dimensiones generales, el proceso de compra, el resultado y la capacidad de recuperación. Así pues, se han considerado como principales medidas de la calidad del servicio de la tienda online, 1) el diseño, organización y facilidad de navegación, 2) cumplimiento y fiabilidad del proceso de compra, 3) la atención al cliente junto a la capacidad de recuperación ante incidencias.

En base a lo indicado anteriormente, en esta investigación se propone la siguiente hipótesis:

- H8: La calidad del servicio online percibida por el consumidor ejerce un efecto directo y positivo sobre la confianza en dicha tienda

\section{Metodología de la investigación}

Para la validación de las hipótesis de esta investigación se procedió a realizar una encuesta sobre compradores en tiendas online. Para ello, se diseñó el instrumento necesario basado en un cuestionario auto-administrado, para medir, desde la perspectiva del consumidor, los diferentes constructos e indicadores, objeto de estudio. Para ello, se utilizaron escalas de medida utilizadas en otras investigaciones o adaptadas, con el objetivo de medir el uso de los medios sociales, 
la notoriedad, la imagen de la tienda, la calidad del servicio ofrecido y la confianza ${ }^{4}$.

De este modo, para la escala de medida de los medios sociales, se utilizaron 6 indicadores de diferentes investigaciones (Gelbrich, 2011; De Villiers et al., 2012; Kim y Ko, 2012), para la medida de la escala de notoriedad, se utilizaron 4 indicadores basados en los trabajos de Aaker (1996), Jarvenpaa et al. (2000) y Pappu y Quester (2006). Para la escala de imagen de la tienda, 4 indicadores basados en las investigaciones de Flavián y Guinaliu (2006) y Calvo-Porral et al. (2013). Por último, para la calidad de servicio se utilizaron 6 indicadores de Wolfinbarger y Gilly (2003), Parasuraman et al. (2005), Collier y Bienstock (2006) y Gil-Saura et al. (2013), mientras que para la medida de la confianza en la tienda online, se utilizaron 4 indicadores de Jarvenpaa et al. (2000), Wolfinbarger y Gilly (2003), Parasuraman et al. (2005), Collier y Bienstock (2006) y Flavián y Guinaliu (2006).

La encuesta final estaba formada por dos áreas, la primera con datos de carácter sociodemográfico y la segunda de carácter cuantitativo formada por 5 bloques y un total de 24 indicadores para las diferentes escalas de medida que eran medidos mediante una escala de Likert de 7 anclajes para favorecer que los datos obtenidos siguiesen una distribución normal y se pudiesen obtener estimaciones robustas, libres de errores tipo I y II, tras la aplicación de técnicas estadísticas multivariantes, ya que un mayor número de anclajes mejora la calidad de la información y aumenta la probabilidad de que la muestra siga una distribución normal y tenga mayor variabilidad (Washburn y Planck, 2002), siendo una forma adecuada de representar intervalos y una

4 Los indicadores utilizados para cada una de las escalas se encuentran en la Tabla 1 buena herramienta para realizar análisis factoriales y contrastes (Hair et al., 2006).

Dada la dificultad de encontrar un censo de compradores de tiendas online, para la obtención de la muestra, se realizó un proceso de muestreo bietápico, donde primero se realizó un muestreo de conveniencia y posteriormente un muestreo de bola de nieve, en línea con otras investigaciones de la literatura (Baltar y Brunet, 2012; Szolnoki y Hoffmann, 2013; Walsh et al., 2016) que sugieren que este procedimiento de muestreo ha mostrado ser eficiente en términos de coste y permite obtener la información en un tiempo menor.

Para ello, se envió un email a un grupo de 500 contactos a los que se les pedía, además de que contestasen a las preguntas del cuestionario electrónico, sobre su experiencia real de compra en tiendas online, que enviasen la encuesta a otros conocidos. La muestra definitiva estaba formada por un total de 301 individuos, todos ellos, compradores habituales de tiendas online y representativos de la población objeto de estudio. De dicho conjunto, por género, 152 eran hombres y 149 mujeres, todos ellos con edades comprendidas entre los 18 y 65 años, con diferentes niveles de formación y diversa actividad profesional. Todo ello buscaba representar lo mejor posible a la población española que compra online. Algunos autores como López y Sicilia (2013) utilizan este tipo de muestreo en sus investigaciones y sugieren que permite aumentar la probabilidad de que la muestra represente a la población objeto de estudio. Además de ello, para analizar la aleatoriedad de la muestra se realizó contraste de rachas de la media y la mediana, dando resultados estadísticamente satisfactorios.

En cuanto al procedimiento de análisis de los datos, se utilizaron técnicas de análisis multivariante basadas en el análisis factorial, confirma- 


\section{Figura 1 - Modelo de ecuaciones estructurales para contraste de hipótesis}

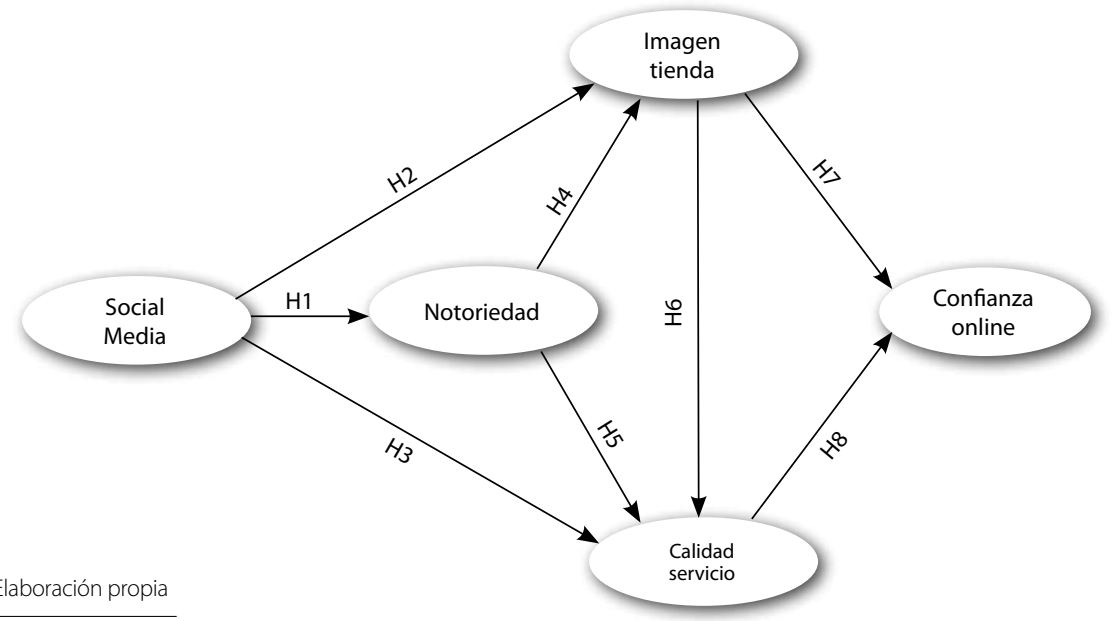

torio para la estimación del modelo de medida y un modelo de ecuaciones estructurales para la estimación de las relaciones causales entre los constructos y el contraste de las hipótesis, tal como el que se observa en la Figura 1. Asimismo, el tamaño de la muestra, cumple con el criterio de Kline (2005) y Hair et al. (2006) al ser de tamaño superior a 200 y el criterio de Bollen (1989) ya que se dispone de 10 observaciones por cada indicador.

El paquete estadístico utilizado fue SPSS y AMOS en su versión 22.0, bajo sistema Windows 7 , siendo el procedimiento de estimación empleado el de máxima verosimilitud por haber mostrado ser bastante robusto en investigaciones similares de otros autores.

\section{Análisis de resultados}

El análisis factorial confirmatorio inicial mostraba, para cada una de las variables de cada uno de los constructos, el valor de la carga estandarizada, así como t-valor, p-valor y R2 individual.
Igualmente, para cada una de las variables latentes se midió la fiabilidad y validez mediante $\alpha$-Cronbach, fiabilidad compuesta (FC) y varianza media extraída (AVE). Todas las medidas se contrastaban con los valores indicados por Hair et al. (2006) y Cea D'Ancona (2002).

Todos los constructos mostraban valores de $\alpha$-Cronbach situados entre 0,726 para la confianza y 0,886 para la calidad del servicio online, superando el límite de 0,7 y confirmando así la fiabilidad de todas las variables latentes.

Dentro del proceso de depuración de las escalas, se eliminaron aquellos indicadores que mostraban valores del coeficiente de determinación individual R2, inferior a 0,5. En esta línea, se procedió a eliminar la variable CAL3 («Entrega de la compra sin errores», R2 = 0,202), NOT4 ( Recuerdo del logosímbolo», R2 = 0,132), ASO4 ( Amigos y Familiares compran en esta tienda», R2 = 0,211), SM1 ( El sitio web permite consultar opiniones de otros», R2=0,097), SM4 («Intención de recomendación en redes 
sociales», R2 = 0,03) y CONF3 ( Privacidad de datos de clientes», $\mathrm{R} 2=0,168$ ). Así pues, el modelo de medida reajustado estaba formado por 18 indicadores y 5 variables latentes.

Tras la eliminación de las citadas variables y el reajuste del modelo, se procedió a realizar un nuevo análisis factorial confirmatorio, con los resultados que se muestran en la Tabla 1.
Como se puede observar, todos los ítems tienen cargas estandarizadas que superan el mínimo de 0,7 , son significativas ( $<<0,001$ ) y tienen valores de R2 individual superior a 0,5. En cuanto a la medida de la fiabilidad, para todos los constructos, $\alpha$-Cronbach y FC superan el límite 0,7 y mejoran la medida de fiabilidad inicial, mientras que AVE supera el valor de 0,5. En base

\section{Tabla 1 - Análisis factorial confirmatorio (modelo de medida) y bondad de ajuste}

\begin{tabular}{|c|c|c|c|c|c|c|c|c|c|}
\hline Construct & & $\begin{array}{l}\text { Indica- } \\
\text { dor }\end{array}$ & $\begin{array}{l}\text { Carga } \\
\text { estan- }\end{array}$ & t-valor & $\mathrm{p}$ & $\mathbf{R}^{2}$ & $\alpha$-Cronbach & $\begin{array}{l}\text { Fiabilidad } \\
\text { Compuesta }\end{array}$ & AVE \\
\hline & Diseño del sitio web & CAL1 & 0,839 & & & 0,704 & 0,910 & 0,912 & 0,676 \\
\hline & Facilidad de navegación & CAL2 & 0,824 & 17,229 & *** & 0,679 & & & \\
\hline $\begin{array}{l}\text { Callada de } \\
\text { servicio }\end{array}$ & Cumplimiento plazos entrega & CAL4 & 0,731 & 14,448 & $* * *$ & 0,534 & & & \\
\hline & Servicio al cliente & CAL5 & 0,816 & 16,973 & $* * *$ & 0,666 & & & \\
\hline & Capacidad de resolución problemas & CAL6 & 0,893 & 19,49 & *** & 0,797 & & & \\
\hline & Grado de conocimiento general de la tienda & NOT1 & 0,919 & & & 0,845 & 0,921 & 0,921 & 0,795 \\
\hline Notoriedad & Recuerdo del nombre de la tienda & NOT2 & 0,870 & 22,024 & $* * *$ & 0,757 & & & \\
\hline & Recuerdo del dominio en internet & NOT3 & 0,886 & 22,778 & $* * *$ & 0,785 & & & \\
\hline Asociacio- & Reputación & ASO1 & 0,805 & & & 0,648 & 0,844 & 0,853 & 0,660 \\
\hline imagen & Experiencia de la tienda & $\mathrm{ASO} 2$ & 0,722 & 13,179 & $* * *$ & 0,521 & & & \\
\hline tienda & Liderazgo & $\mathrm{ASO} 3$ & 0,901 & 16,404 & $* * *$ & 0,812 & & & \\
\hline & Inspira confianza & CONF1 & 0,787 & & & 0,619 & 0,787 & 0,792 & 0,559 \\
\hline Confianza & Cumplimiento de los compromisos & CONF2 & 0,729 & 11,501 & $* * *$ & 0,531 & & & \\
\hline & Seguridad & CONF4 & 0,726 & 11,466 & $* * *$ & 0,527 & & & \\
\hline & Presencia en redes sociales & SM2 & 0,825 & & & 0,681 & 0,912 & 0,913 & 0,724 \\
\hline Social & Interés por comentarios y recomendaciones & SM3 & 0,860 & 17,815 & $* * *$ & 0,740 & & & \\
\hline media & $\begin{array}{l}\text { Influencia de comentarios en decisión de } \\
\text { compra }\end{array}$ & SM5 & 0,813 & 16,406 & $* * *$ & 0,661 & & & \\
\hline & Los comentarios son beneficiosos & SM6 & 0,903 & 19,009 & $* * *$ & 0,815 & & & \\
\hline & & & & dices de & ajuste c & al mod & & & \\
\hline$x^{2}$ & & GFI & TLI & RFI & NFI & grado & de libertad & $=125 * * *$ & $p<0,001$ \\
\hline 325,642 & & 0,894 & 0,933 & 0,895 & 0,914 & & & & \\
\hline$x^{2 / g l}$ & & IFI & CFI & AGFI & RMSEA & parán & etros $=46$ & & \\
\hline 2,605 & & 0,945 & 0,945 & 0,855 & 0,073 & & & & \\
\hline
\end{tabular}


a ello, se confirma la validez convergente y la fiabilidad de los constructos del modelo.

En cuanto al ajuste del modelo de medida, los indicadores alcanzaban valores satisfactorios de acuerdo con la literatura, destacando el valor del indicador CFI superior a 0,9 así como el valor de $\chi 2 /$ gl cercano a 2 y RMSEA inferior a 0,08 lo que indica un ajuste satisfactorio.

A continuación, se procedió a determinar la validez discriminante (Cea D'Ancona, 2002; Hair et al., 2006) de los constructos para ello se realizó, por un lado, el test de la raíz cuadrada del AVE y, por otro, la prueba del intervalo de confianza, tal y como se muestra en la Tabla 2. Los resultados confirman la validez discriminante de todos los constructos.

En cuanto a las relaciones causales entre los constructos, se realizó un análisis multivariante basado en ecuaciones estructurales, dando como resultado lo que se observa en la Tabla 3.

A la vista de estos resultados, se puede confirmar que el proceso de notoriedad para la tienda online está directa y positivamente influenciado por los medios sociales que facilita el minorista $(\beta=0,357)$, con un nivel de significación ( $\mathrm{p}<$ $0,001)$ y, por tanto, se acepta la hipótesis H1.

Igualmente, el proceso de generación de imagen de la tienda online, está directa y positivamente influenciado por la notoriedad $(\beta=0,610)$ con un nivel de significación ( $\mathrm{p}<0,001)$ y por los medios sociales $(\beta=0,116)$ con un nivel de significación ( $p<0,05)$, de modo que, se aceptan las hipótesis $\mathrm{H} 2$ y H3.

En cuanto al constructo de calidad de servicio online, se encuentra directa y positivamente influenciado por la imagen de la tienda $(\beta=0,345)$

\section{Tabla 2 - Validez discriminante (Intervalo de confianza y Test AVE)}

\begin{tabular}{|l|c|c|c|c|c|}
\hline & SM & Notoriedad & Imagen & \multicolumn{1}{c|}{ Calidad } & Confianza \\
\hline SM & $\mathbf{0 , 8 5 1}$ & $(0,284-0,430)$ & $(0,276-0,402)$ & $(0,078-0,204)$ & $(0,054-0,160)$ \\
\hline Notoriedad & 0,357 & $\mathbf{0 , 8 9 2}$ & $(0,601-0,709)$ & $(0,150-0,244)$ & $(0,259-0,341)$ \\
\hline Imagen & 0,339 & 0,655 & $\mathbf{0 , 8 1 3}$ & $(0,287-0,373)$ & $(0,500-0,580)$ \\
\hline Calidad & 0,141 & 0,197 & 0,330 & $\mathbf{0 , 8 2 2}$ & $(0,545-0,633)$ \\
\hline Confianza & 0,107 & 0,300 & 0,540 & 0,589 & $\mathbf{0 , 7 4 8}$ \\
\hline
\end{tabular}

Fuente: Elaboración propia

Tabla 3 - Análisis de relaciones causales (Modelo estructural - SEM), estimación y contraste de hipótesis

\begin{tabular}{|c|l|c|c|c|c|}
\hline Hipótesis & Descripción & $\begin{array}{c}\text { Estimación } \\
\text { estandarizada }\end{array}$ & T-Valor & Resultado \\
\hline H1 & Medios Sociales - Notoriedad & 0,357 & 5,831 & $* * *$ \\
\hline H2 & Medios Sociales - Imagen de la Tienda & 0,116 & 2,065 & 0,039 \\
\hline H3 & Medios Sociales - Calidad de Servicio & 0,036 & 0,555 & 0,579 \\
\hline H4 & Notoriedad - Imagen de la Tienda & 0,61 & 9,652 & Aceptada \\
\hline H5 & Notoriedad - Calidad de Servicio & $-0,042$ & $-0,492$ & 0,623 \\
\hline H6 & Imagen de la Tienda - Calidad de Servicio & 0,345 & 3,858 & Aceptada \\
\hline H7 & Imagen de la Tienda - Confianza & 0,386 & 5,744 & Rechazada \\
\hline H8 & Calidad de Servicio - Confianza & 0,482 & 7,16 & Aceptada \\
\hline Fuente: Elaboración propia & Aceptada & $* * *$ \\
\hline
\end{tabular}


con un nivel de significación $(p<0,001)$. Sin embargo, no existe relación significativa entre los medios sociales o la notoriedad con la calidad del servicio online. En base a dichos resultados, se acepta la hipótesis H6 y se rechazan las hipótesis $\mathrm{H} 3$ y $\mathrm{H} 5$.

Por último, la confianza en la tienda online viene influenciada directa y positivamente tanto por la imagen de la tienda en la mente del consumidor $(\beta=0,386)$ como por la calidad de servicio online percibida $(\beta=0,482)$, ambos casos con niveles de significación ( $p<0,001)$. Asimismo, la confianza en la tienda online, a través de las relaciones causales del modelo, se encuentra explicada en un 52\%, tal y como indica coeficiente de determinación R2. En base a estos resultados, se procede a aceptar las hipótesis $\mathrm{H} 7 \mathrm{y} \mathrm{H8}$.

Por último, en cuanto al encaje global del modelo estructural, todos los indicadores eran satisfactorios y alcanzaban los mínimos exigidos por la literatura, tal y como se observa en la Tabla 4. Respecto del modelo confirmatorio, ha habido una mejora en la parsimonia, tal y como se observa en el valor de $\chi 2 / g l$.

Así pues, a la vista de dichos resultados, el modelo estructural de relaciones causales, quedó tal y como se observa en la Figura 2.

\section{Tabla 4 • Índices de bondad de ajuste del modelo estructural}

\begin{tabular}{|c|c|c|c|c|c|}
\hline & \multicolumn{5}{|c|}{ Índices de ajuste del modelo } \\
\hline $\mathbf{X} 2$ & GFI & TLI & RFI & NFI & grados de libertad = 127 \\
\hline 329,005 & 0,893 & 0,933 & 0,896 & 0,914 & \\
\hline $\mathbf{X} 2 / \mathbf{g l}$ & IFI & CFI & AGFI & RMSEA & parámetros $=44$ \\
\hline 2,591 & 0,945 & 0,945 & 0,856 & 0,073 & $* * * 0<001$ \\
\hline Fuente: Elaboración propia & & & & \\
\hline
\end{tabular}

\section{Figura 2 - Modelo estructural y estimación de relaciones causales}

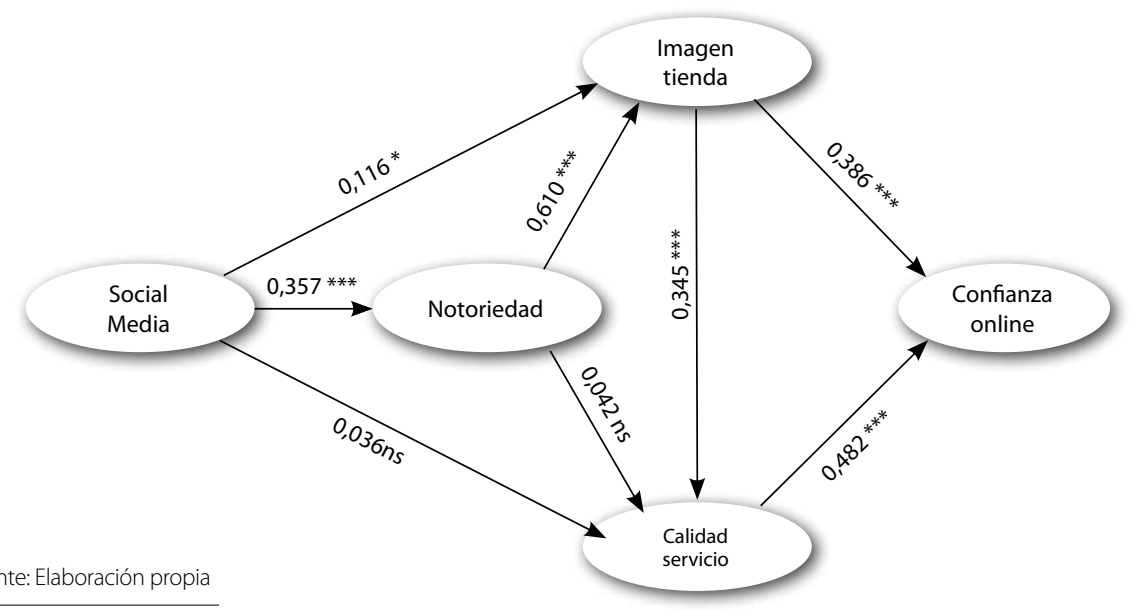




\section{Conclusiones, limitaciones y futuras líneas de investigación}

A la vista de los resultados obtenidos de la investigación empírica, la confianza del consumidor en una tienda online viene determinada, de forma directa, por la imagen y asociaciones que tiene de la misma así como la percepción de calidad de servicio que ofrece el establecimiento. Por ello, los directivos de las tiendas online, deberían dedicar recursos para mejorar, tanto la reputación como proyectar a los consumidores un elevado grado de experiencia en el comercio electrónico y buscar ser, al menos en la mente del consumidor, el líder del sector o de su categoría. De igual forma, se debería reforzar el proceso de generación de calidad de servicio, centrando los esfuerzos, tanto en el diseño y facilidad de navegación por el sitio web, como el cumplimiento de los plazos de entrega acordados así como el desarrollo de un servicio posventa que sea capaz de llevar a cabo una atención al cliente, eficaz y que de respuesta rápida a las incidencias de los clientes.

En cuanto a la presencia en los medios sociales, parece relevante la presencia en las distintas redes sociales y que el minorista online favorezca el desarrollo de comunidades virtuales donde los clientes puedan aportar sus comentarios y recomendaciones acerca de la tienda, lo que tendrá un mayor efecto sobre el grado de conocimiento general de la misma así como un efecto sobre la imagen que proyecta sobre los consumidores. Una mejora de la imagen percibida de la tienda podría influenciar positivamente la percepción de calidad de servicio online, la cual, a la vista de los resultados, no estaría influenciada por las actividades desarrolladas en los medios sociales.

Por otro lado, esta investigación está sujeta a una serie de limitaciones que deberían ser abordadas en futuros estudios. En primer lugar, el tamaño de la muestra, si bien es suficiente desde el punto de vista estadístico, debería ser mayor para conseguir una aproximación mejor al universo objeto de estudio.

Igualmente, la utilización de escalas probadas en la literatura sobre establecimientos minoristas, podrían esconder la ausencia de otras variables de interés, ya que el comercio electrónico es un concepto dinámico que día a día conlleva cambios en las percepciones de los consumidores. Por ello, una limitación posible sería la derivada del desarrollo de escalas para los constructos, más actuales y más adecuadas a los nuevos entornos online y las nuevas tecnologías.

Por último, la segmentación en la muestra y en la investigación, al menos por género, podría dar como resultado, diferentes modelos estructurales y de medida, algo en lo que se debería profundizar en futuras investigaciones

Así pues, de acuerdo con la literatura existente sobre tiendas online, la confianza parece ser un factor determinante en la intención de compra y sus principales dimensiones son, de acuerdo con los resultados de la investigación, la imagen que el consumidor tiene de la tienda online así como la calidad del servicio, de forma directa y, la información o comentarios de los medios sociales y la notoriedad de la tienda, de forma indirecta. 


\section{Bibliografía}

Aaker, D. A. (1996). Measuring brand equity across products and markets. California Management Review, 38(3), 102-120.

Aladwani, A. M. (2006). An empirical test of the link between web site quality and forward enterprise integration with web consumers. Business Process Management Journal, 12(2), 178-190.

Arce, M., y Cebollada, J. (2011). Una comparación del comportamiento del consumidor en los canales online y offline: sensibilidad al precio, lealtad de marca y efecto de las características del producto. Cuadernos de Economía y Dirección de la Empresa (14), 102-111.

Ayestarán, R. (2014). Social Media como canal estratégico y operativo dentro del medio digital. Revista Internacional de Investigación en Comunicación aDResearch ESIC, 10(10), 126-153.

Balaghi, M. (2014). Investigation of website brand equity on online shopping behavior. Journal of Applied Sciences Research, 10(5), 590-597.

Baltar, F., y Brunet, I. (2012). Social research 2.0: virtual snowball sampling method using Facebook. Internet Research, 22(1), 57-74.

Bart, Y., Shankar, V., Sultan, F., y Urban, G. L. (2005). Are the drivers and role of online trust the same for all web sites and consumers? A large-scale exploratory empirical study. Journal of Marketing, 69(4), 133-152.

Bazarova, N. N. (2016). Sharing our lives online: Risks and exposure in social media. Journal of Broadcasting and Electronic Media, 60(1), 190-192.

Beristain, J. J., y Zorrilla, P. (2011). The relationship between store image and store brand equity: A conceptual framework and evidence from hypermarkets. Journal of Retailing and Consumer Services, 18(6), 562-574.

Berry, L.; Seiders, K. y Grewal, D. (2002) Understanding service convenience. Journal of Marketing, 66(3), 1-17.

Bollen, K. A. (1989). Structural equations with latent variables. New York, NY: Wiley.

Brown, J., Broderick, A. J., y Lee, N. (2007). Word of mouth communication within online communities: Conceptualizing the online social network. Journal of Interactive Marketing, 21(3), 2-20.
Calvo-Porral, C., Martinez-Fernández, V. A., Juanatey-Boga, O., y Lévy-Mangín, J. P. (2013). What matters to store brand equity? An approach to Spanish large retailing in a downturn context. Investigaciones Europeas de Dirección y Economía de la Empresa, 19(3), 136-146.

Casalo, L. V., Flavián, C., y Guinalíu, M. (2007). The influence of satisfaction, perceived reputation and trust on a consumer's commitment to a website. Journal of Marketing Communications, 13(1), 1-17.

Castelló, A. (2010). ¿Cuánto vale un fan?. El reto de la medición de la audiencia en los social media. Pensar en Publicidad, 4(2), 89-110.

Cea D’Ancona, M. A. (2002). Análisis multivariable: teoría y práctica en la investigación social. Madrid: Sintesis.

Chen, L. S. (2012). What drives cyber shop brand equity? An empirical evaluation of online shopping system benefit with brand experience. International Journal of Business and Information, 7(1), 81-104.

Cheung, C. M., Lee, M. K., y Rabjohn, N. (2008). The adoption of online opinions in online customer communities. Internet Research, 18(3), 229-247.

Collier, J. E., y Bienstock, C. C. (2006). Measuring service quality in e-retailing. Journal of Service Research, 8(3), 260275 .

Constantinides, E. (2004). Influencing the online consumer's behavior: the Web experience. Internet Research, 14(2), 111-126.

De Salas, M.I. (2002). Internet como campo para el desarrolo de nuevas formas de publicidad. Area abierta. № 4 .

De Ruyter, K., Wetzels, M., y Kleijnen, M. (2001). Customer adoption of e-service: an experimental study. International Journal of Service Industry Management, 12(2), 184-207.

De Villiers, R., Donaldson, J., Priilaid, D., y Watson, N. (2012). Factors driving online brand equity in an emerging market: A South African Case Study. SAIMS 2013. Conference Paper, 283-308.

De Vries, L., Gensler, S., y Leeflang, P. S. (2012). Popularity of brand posts on brand fan pages: An investigation of the effects of social media marketing. Journal of Interactive Marketing, 26(2), 83-91.

Dellarocas, C., y Narayan, R. (2006). A statistical measure of a population's propensity to engage in post-purchase online word-of-mouth. Statistical Science, 21(2), 277-285. 
Dick, A., Jain, A., y Richardson, P. (1995). Correlates of store brand proneness: some empirical observations. Journal of Product and Brand Management, 4(4), 15-22.

Dijkmans, C., Kerkhof, P., y Beukeboom, C. (2015). A stage to engage: Social media use and corporate reputation. Tourism Management, 47, 58-67.

Doherty, N.; Ellis-Chadwick, F. y Hart, C.A. (2003). An analysis of the factors affecting the adoption of the internet in the UK retail sector. Journal of Business Research, 56 (11), 887-897.

Dong-Her, S., Hsiu-Sen, C., Chun-Yuan, C., y Lin, B. (2004). Internet security: malicious e-mails detection and protection. Industrial Management and Data Systems, 104(7), 613-623.

Drèze, X., y Zufryden, F. (2004). Measurement of online visibility and its impact on Internet traffic. Journal of Interactive Marketing, 18(1), 20-37.

eMarketer. (2014). eMarketer.com. ultimo acceso 10 de octubre de 2015, http://www.emarketer.com/Article/InternetHit-3-Billion-Users-2015/1011602

Fanion, R. (2011). Social Media brings benefits to top companies. Central Penn Business Journal, 27(33), 76-77.

Flavián, C., y Guinaliu, M. (2006). Consumer trust, perceived security and privacy policy: Tree basic elements of loyalty to a web site. Industrial Management and Data Systems, 106(5), 601-620.

Forsythe, S., y Shi, B. (2003). Consumer patronage and risk perceptions in Internet shopping. Journal of Business Research, 56(11), 867-875.

Gefen, D., y Straub, D. W. (2004). Consumer trust in B2C e-Commerce and the importance of social presence: experiments in e-Products and e-Services. Omega, 32(6), $407-$ 424.

Gefen, D., Karahanna, E., y Straub, D. W. (2003). Trust and TAM in online shopping: an integrated model. MIS quarterly, 27(1), 51-90.

Gelbrich, K. (2011). I have paid less than you! The emotional and behavioral consequences of advantaged price inequality. Journal of Retailing, 87(2), 207-224.

Gil-Saura, I., Ruiz-Molina, M. E., Michel, G., y CorralizaZapata, A. (2013). Retail brand equity: a model based on its dimensions and effects. The International Review of Retail, Distribution and Consumer Research, 23(2), 111-136.
Goode, M. y Harris, L. (2007). Online behavioural intentions: an empirical investigation of antecedents and moderators. European Journal of Marketing, 41(5/6), 512-536.

Golob, U., Podnar, K., Elving, W. J., Ellerup, A., y Thomsen, C. (2013). CSR communication: Quo vadis? Corporate Communications: An International Journal, 18(2), 176192.

Grewal, D., Baker, J., Levy, M., y Voss, G. B. (2003). The effects of wait expectations and store atmosphere evaluations on patronage intentions in service-intensive retail stores. Journal of Retailing, 79(4), 259-268.

Gruen, T.W., Osmonbekov, T., y Czaplewski, A.J. (2006). eWOM: The impact of customer-to customer online knowhow exchange on customer value and loyalty. Journal of Business Research, 59(4), 449-456.

Gunelius, S. (2011). 30-minute social media marketing. New York, NY: McGraw-Hill.

Hair, J. F., Black, W. C., Babin, B. J., Anderson, R. E., y Tatham, R. L. (2006). Multivariate data analysis (Ed. 6). Upper Saddle River, NJ: Pearson Prentice Hall.

Haque, A., Momen, A., Sultana, S., y Yasmin, F. (2013). Online brand awareness: determining the relative importance of facebook and other strategies among the malaysian consumers. Information Management and Business Review, 5(4), 168-174.

Hartman, K.B., y Spiro, R.S. (2005). Recapturing store image in consumer-based store equity: a

construct conceptualization. Journal of Business Research, 58(8), 1112-1120.

Holland, J., y Baker, S. M. (2001). Customer participation in creating site brand loyalty. Journal of Interactive Marketing, 15(4), 34-45.

Hsieh, Y. C., Roan, J., Pant, A., Hsieh, J. K., Chen, W. Y., Lee, M., y Chiu, H. C. (2012). All for one but does one strategy work for all? Building consumer loyalty in multichannel distribution. Managing Service Quality: An International Journal, 22(3), 310-335.

Ioan s, E., y Stoica, I. (2014). Social media and its impact on consumers behavior. International Journal of Economic Practices and Theories, 4(2), 295-303.

Jarvenpaa, S., Tractinsky, N., y Vitale, M. (2000). Consumer trust in Internet stores. Information Technology and Management, 1(1-2), 45-71. 
Kaplan, A. M., y Haenlein, M. (2010). Users of the world, unite! The challenges and opportunities of Social Media. Business Horizons, 53(1), 59-68.

Keller, K. L. (1993). Conceptualizing, measuring and managing customer-based brand equity. Journal of Marketing, 57(1), 1-22.

Khan, N., Rahmani, S. H., Hoe, H. Y., y Chen, T. B. (2015). Causal relationships among dimensions of consumer-based brand equity and purchase intention: Fashion Industry. International Journal of Business and Management, 10(1), 172-181.

Kietzmann, J. H., Hermkens, K., McCarthy, I. P., y Silvestre, B. S. (2011). Social media? Get serious! Understanding the functional building blocks of social media. Business Horizons, 54(3), 241-251.

Kim, A. J., y Ko, E. (2012). Do social media marketing activities enhance customer equity? An empirical study of luxury fashion brand. Journal of Business Research, 65(10), 1480-1486.

Kim, J., y Forsythe, S. (2010). Factors affecting adoption of product virtualization technology for online consumer electronics shopping. International Journal of Retail and Distribution Management, 38(3), 190-204.

Kim, J., Jin, B., y Swinney, J. L. (2009). The role of retail quality, e-satisfaction and e-trust in online loyalty development process. Journal of Retailing and Consumer Services, 16(4), 239-247.

Kline, T. J. (2005). Psychological testing: A practical approach to design and evaluation. Thousand Oaks, CA, USA: Sage Publications.

Kotler, P., y Keller, K. L. (2012). Dirección de marketing, 14th Ed. Naucalpan de Juárez, Mexico: Pearson Educación.

Laroche, M., Habibi, M. R., y Richard, M. O. (2013). To be or not to be in social media: How brand loyalty is affected by social media? International Journal of Information Management, 33(1), 76-82.

Laroche, M., Habibi, M. R., Richard, M. O., y Sankaranarayanan, R. (2012). The effects of social media based brand communities on brand community markers, value creation practices, brand trust and brand loyalty. Computers in $\mathrm{Hu}$ man Behavior, 28(5), 1755-1767.

Lee, G. G., y Lin, H. F. (2005). Customer perceptions of eservice quality in online shopping. International Journal of Retail and Distribution Management, 33(2), 161-176.
Liberos, E., del Poyo, R. G., Rabadán, J. G., Merino, J. A., y Somalo, I. (2010). El libro del comercio electrónico. Madrid: ESIC.

López, M., y Sicilia, M. (2013). Boca a boca tradicional vs electrónico. La participación como factor explicativo de la influencia del boca a boca electrónico. Revista Española de Investigación en Marketing ESIC, 17(1), 7-38.

Manganari, E. E., Siomkos, G. J., y Vrechopoulos, A. P. (2009). Store atmosphere in web retailing. European Journal of Marketing, 43(9/10), 1140-1153.

Mangold, W. G., y Faulds, D. J. (2009). Social media: The new hybrid element of the promotion mix. Business Horizons, 52(4), 357-365.

Marsden, P. (2011). Commerce gets social: How your networks are driving what you buy. Social Commerce Today, $1-10$.

Martenson, R. (2007). Corporate brand image, satisfaction and store loyalty: A study of the store as a brand, store brands and manufacturer brands. International Journal of Retail and Distribution Management, 35(7), 544-555.

Martínez, F. J., Ortigueira, M., y Pérez, M. (2006). Confianza del consumidor en la compra a través de Internet: una propuesta de modelización basada en la jerarquía de aprendizaje estándar. Cuadernos de Gestión, 6(2), 59-82.

Neslin, S. A., Grewal, D., Leghorn, R., Shankar, V., Teerling, M. L., Thomas, J. S., y Verhoef, P. C. (2006). Challenges and opportunities in multichannel customer management. Journal of Service Research, 9(2), 95-112.

ONTSI. (2015). Estudio sobre el Comercio Electrónico B2C 2014. Observatorio Nacional de las Telecomunicaciones y la Sociedad de la Información - Red.es., último acceso 25 noviembre de 2015, http://www.ontsi.red.es/ontsi/es/estudios-informes/estudio-b2c-2014-edici\%C3\%B3n-2015

Page, C. y Lepkowska-White, E. (2002). Web equity: A framework for building consumer value in online companies. Journal of Consumer Marketing, 19(3), 231-246.

Pantano, E., y Viassone, M. (2015). Engaging consumers on new integrated multichannel retail settings: Challenges for retailers. Journal of Retailing and Consumer Services, 25, 106-114.

Pappu, R., y Quester, P. (2006). A consumer-based method for retailer equity measurement: Results of an empirical study. Journal of Retailing and Consumer Services, 13(5), 317-329. 
Pappu, R., Quester, P. G., y Cooksey, R. W. (2005). Consumer-based brand equity: improving the measurement-empirical evidence. Journal of Product and Brand Management, 14(3), 143-154.

Parasuraman, A., Zeithaml, V. A., y Berry, L. L. (1988). Journal of Retailing, 64(1), 12-40.

Parasuraman, A., Zeithaml, V. A., y Malhotra, A. (2005). A multiple-item scale for assessing electronic service quality. Journal of Service Research, 7(3), 213-233.

Park, D.H.; Lee, J., y Han, I. (2007). The effect of on-line consumer reviews on consumer purchasing intention: The moderating role of involvement. International Journal of Electronic Commerce, 11(4), 125-148.

Park, J. S., y Fairhurst, A. (2010). Consumer perception of social presence in e-tail websites. Journal of the Korean Society of Clothing and Textiles, 34(6), 997-1007.

Pavlou, P. A. (2003). Consumer acceptance of electronic commerce: Integrating trust and risk with the technology acceptance model. International Journal of Electronic Commerce, 7(3), 101-134.

Prado, A., Blanco, A., y Mercado, C. (2014). Análisis del proceso de generación de lealtad en el entorno on-line a través de la calidad del servicio y la calidad de la relación. Revista Europea de Dirección y Economía de la Empresa, 23, 175-183.

Quelch, J., y Klein, L. (1996). The Internet and international marketing. MIT Sloan Management Review, 37(3), 60-75.

Ribbink, D., Van Riel, A. C., Liljander, V., y Streukens, S. (2004). Comfort your online customer: Quality, trust and loyalty on the Internet. Managing Service Quality: An International Journal, 14(6), 446-456.

Rodríguez, I. (2008). Marketing.com y comercio electrónico en la sociedad de la información (Ed. $3^{\mathrm{a}}$ ). Madrid: Ediciones Pirámide.

Rowley, J. (2006). An analysis of the e-service literature: towards a research agenda. Internet Research, 16(3), 339359.

Serrano-Cinca, C., Fuertes-Callén, Y., y Gutiérrez-Nieto, B. (2010). Internet positioning and performance of e-tailers: An empirical analysis. Electronic Commerce Research and Applications, 9(3), 237-248.

Sethuraman, R.; Tellis, G. y Briesch, R. (2011) How well does advertising work? Generalizations from meta-analysis of brand advertising elasticities. Journal of Marketing Research, 48(3), 457-471.

Shojaee, S., y Azman, A. B. (2013). An evaluation of factors affecting brand awareness in the context of social media in Malaysia. Asian Social Science, 9(17), 72-78.

Silverstein, B. (2002). Business to business Internet Marketing. Gulf Breeze, Florida: Maximmum Press.

Strater, K., y Richter, H. (2007). Examining privacy and disclosure in a social networking community. Proceedings of the 3rd symposium on Usable privacy and security (pp. 157-158). Pittsburgh, PA: ACM.

Szolnoki, G., y Hoffmann, D. (2013). Online, face-to-face and telephone surveys-Comparing different sampling methods in wine consumer research. Wine Economics and Policy, 2(2), 57-66.

Tresna, L., y Wijaya, J. C. (2015). The impact of social media towards brand equity: an empirical study of mall $\mathrm{x}$. iBuss Management, 3(2), 37-48.

Trusov, M.; Bucklin, R., y Pauwels K. (2009). Effects of word-of-mouth versus traditional marketing: Findings from an Internet social networking site. Journal of Marketing, 73(5), 90-102.

Tsimonis, G., y Dimitriadis, S. (2014). Brand strategies in social media. Marketing Intelligence and Planning, 32(3), 328-344.

Urban, G. L., Sultan, F, y Quails, W. J. (2000). Placing trust at the center of your Internet strategy. MIT Sloan Management Review, 42(1), 39-48.

Villarejo-Ramos, Á. F., Sánchez-Franco, M. J., y Cataluña, F. R. (2007). Antecedentes de la notoriedad del nombre en la determinación de la imagen de marca: una visión desde un producto de gran consumo. En El comportamiento de la empresa ante entornos dinámicos. XIX Congreso anual y XV Congreso Hispano Francés de AEDEM (pp. 1-10). Vitoria-Gasteiz: Asociación Española de Dirección y Economía de la Empresa (AEDEM).

Vrechopoulos, A. P., O'Keefe, R. M., Doukidis, G. I., y Siomkos, G. J. (2004). Virtual store layout: an experimental comparison in the context of grocery retail. Journal of Retailing, 80(1), 13-22.

Walsh, G., Albrecht, A. K., Hofacker, C. F., Grant, I., y Takahashi, I. (2016). Developing and validating a scale of consumer brand embarrassment tendencies. Journal of Business Research, 69(3), 1138-1147. 
Wang, C. C., y Hwang, I. S. (2001). The influence of product knowledge on the amount of merchandises information search on Internet. Journal of Business Administration, 51, 109-138.

Washburn, J. H., y Plank, R. E. (2002). Measuring brand equity: An evaluation of a consumer-based brand equity scale. Journal of Marketing Theory and Practice, 10(1), 46-62.

Wolfinbarger, M., y Gilly, M. C. (2003). eTailQ: dimensionalizing, measuring and predicting etail quality. Journal of Retailing, 79(3), 183-198.

Wu, J. J., y Chang, Y. S. (2006). Effect of transaction trust on e-commerce relationships between travel agencies. Tourism Management, 27(6), 1253-1261.

Wu, W. Y., Lee, C. L., Fu, C. S., y Wang, H. C. (2013). How can online store layout design and atmosphere influence consumer shopping intention on a website? International Journal of Retail and Distribution Management, 42(1), 4-24.
Yoo, B., y Donthu, N. (2001). Developing a scale to measure the perceived quality of an Internet shopping site (SITEQUAL). Quaterly Journal of Electronic Commerce, 2(1), 31-46.

Yoo, B., Donthu, N., y Lee, S. (2000). An examination of selected marketing mix elements and brand equity. Journal of the Academy of Marketing Science, 28(2), 195-211.

Zhang, J.; Farris, P.; Irvin, J.; Kushwaha, T.; Steenburgh, T. y Weitz, B. (2010). Crafting integrated multichannel retailing strategies. Journal of Interactive Marketing, 24(2), 168-180.

Zeithaml, V. A., Parasuraman, A., y Malhotra, A. (2002). Service quality delivery through web sites: a critical review of extant knowledge. Journal of the Academy of Marketing Science, 30(4), 362-375.

Zhu, F., y Zhang, X. (2010). Impact of online consumer reviews on sales: The moderating role of product and consumer characteristics. Journal of Marketing, 74(2), 133-148. 
